\title{
Prevalence and variability of use of home mechanical ventilators, positive airway pressure and oxygen devices in the Lombardy region, Italy
}

\author{
Michele Vitacca1, Luca Barbano', Daniele Colombo2, Olivia Leoni33, Enrico Guffanti2 \\ 1 U.O. Pneumologia Riabilitativa Istituti Clinici Scientifici Maugeri, IRCCS Lumezzane \\ 2 U.O. Pneumologia Riabilitativa INRCA, IRCCS Casatenovo \\ 3 U.O. Programmazione Polo Ospedaliero Struttura Farmaco, Dispositivi e HTA, Farmacovigilanza, \\ Direzione Generale Welfare, Regione Lombardia, Italy
}

\begin{abstract}
Few studies have analyzed the prevalence and accessibility of home mechanical ventilation (HMV) in Italy. We aimed to investigate the prevalence and prescription variability of HMV as well as of long-term oxygen therapy (LTOT) and continuous positive airway pressure (CPAP), in the Lombardy Region. Prescribing rates of HMV (both noninvasive and tracheostomies), CPAP (auto-CPAP, CPAP/other sleep machines) and LTOT (liquid- $0_{2}, 0_{2}$ gas, concentrators) in the 15 Local Healthcare districts of Lombardy were gathered from billing data for 2012 and compared. Crude rates (per 100,000 population) and rates for
\end{abstract}

Corresponding Author: Michele Vitacca, Istituti Clinici Scientifici Maugeri, IRCCS Lumezzane, U.0. Pneumologia Riabilitativa, Via G. Mazzini 129, 25065 Lumezzane (BS), Italy. Tel. +39.30.8253182 - Fax: +39.30.8253188. E-mail: michele.vitacca@icsmaugeri.it

Keywords: Chronic respiratory failure; noninvasive ventilation; chronic care; home care, oxygen prescription; continuous positive airway pressure.

Contributions: MV, LB, literature search, study design, data analysis, manuscript preparation. All Authors contributed to data collection, manuscript critical evaluation for intellectual content, and revised and approved the final version of the manuscript.

Conflict of interests: The Authors declare that they have no conflict of interests.

Funding: This work was supported by Institutional funding.

Acknowledgements: We thank Alma Lisa Rivolta of Lombardy Data Inspection Board, all the Lombardy Health district Directors for the precious data and information obtained from their Administrative units. We thank Laura Comini for critical revision and editing assistance, and Rosemary Allpress for the English revision.

Received for publication: 16 August 2017

Accepted for publication: 17 December 2017

(C) Copyright M. Vitacca et al., 2018

Tipografia PI-ME Editrice, Italy

Monaldi Archives for Chest Disease 2018; 88:882

doi: 10.4081/monaldi.2018.882

This article is distributed under the terms of the Creative Commons Attribution Noncommercial License (by-nc 4.0) which permits any noncommercial use, distribution, and reproduction in any medium, provided the original author(s) and source are credited. the different healthcare districts were calculated. In 2012, 6325 patients were on HMV (crude prescription rate: $63 / 100,000$ ) with a high variation across districts $(8 / 100,000$ in Milano 1 vs $150 / 100,000$ in Pavia). There were 14,237 patients on CPAP (crude prescription rate: 142/100,000; CPAP/other sleep machines $95.3 \%$ vs auto-CPAP 4.7\%) with also high intra-regional variation $(56 / 100,000$ in Mantova vs. 260/100,000 in Pavia). There were 21,826 patients on LTOT (prescription rate: $217 / 100,000$ rate; liquid- $0_{2} 94 \%, O_{2}$ gas $2.08 \%, 0_{2}$-concentrators $3.8 \%)$, with again high intra-regional variation $(100 / 100,000$ in Bergamo vs 410/100,000 in Valle Camonica). The crude rate of HMV prescriptions in Lombardy is very high, with a high intra-regional variability in prescribing HMV, LTOT and CPAP which is partly explainable by the accessibility to specialist centers with HMV/sleep-study facilities. Analysis of administrative data and variability mapping can help identify areas of reduced access for an improved standardization of services. An audit among Health Payer and prescribers to interpret the described huge variability could be welcomed.

\section{Introduction}

Long-term home mechanical ventilation (HMV) is increasingly being prescribed for people with chronic respiratory failure (CRF) arising from the advanced state of diseases such as chronic obstructive pulmonary disease (COPD), restrictive thoracic disease (RTD) and neuromuscular diseases (NMD). The last reported prevalence (though probably underestimated) of European patients requiring HMV is 6.6/100,000 population [1]. Although HMV has been shown to reduce patients' symptoms, improve health-related quality of life (HRQL) and, in many cases, reduce mortality and hospitalizations [2,3] there are problems connected with providing HMV, such as inadequate patient/caregiver training and lack of compliance, dis-homogeneous reimbursement policies and insufficient resources [4]. Differences in the availability of HMV, and in reimbursement regarding evidencebased medical policies/indications, may also lead to wide variations in the prevalence and the patterns of HMV provision throughout Europe [1,5]. The number of respiratory units and specialists available in a particular area can also account for the variability in prescription, as has been observed in other respiratory therapies such as home oxygen therapy [6]. Despite the growing practice of HMV, few studies have analyzed the prevalence of home respiratory devices prescription, e.g. ventilators, long-term oxygen therapy (LTOT), and continuous positive airway pressure (CPAP) devices.

Variability in the clinical practice regarding HMV is widely recognized and has been the object of study for over 30 years [7]. Decisionmaking is a complex issue, and clinical practice is established taking into account not only scientific evidence but also local circumstances, professional skills and, above all, patient values [8]. The magnitude of 
variability must be studied in order to introduce specific actions for improvement, and to guarantee equal access to effective treatments. A systematic study of variability can be done through disease registers over time, in order to observe trends, or by dedicated surveys addressed to health teams involved in this field. Another possibility is to collect information from national or regional healthcare administrative databases.

HMV started in an important Italian Region (Lombardy) riding between the 80 s and 90 s being home care management a new and pioneering approach for chronic ventilated patients (80\% obstructive and $20 \%$ restrictive/neuromuscular patients) [9]. During the last 3 decades, HMV is dramatically increase also in this Region with inevitable changes in technology, habits, number of prescribers, diseases prescription and costs.

To this end, a dedicated Task Force comprising pulmonologists and healthcare administration staff based in an important Italian Region (Lombardy) carried out such a search of administrative data of the Local Health Authorities in Lombardy in order to characterize the prevalence of HMV, CPAP and LTOT and the variability in prescriptions in Lombardy.

\section{Materials and Methods}

As only administrative data were involved, no ethical approval was required. A web-based observational database was reviewed. This study compared the HMV prescription rates in the different Local Health Authority districts of Lombardy (Italy) active at the time of the survey. Patients were identified using the Lombardy Health Service billing database selecting all cases in which HMV was invoiced for the year 2012. The data collected were anonymous. Cases were assigned to each geographical area according to each patient's healthcare zone of residence. The denominators were calculated using population data from the Lombardy bureau of statistics (ISTAT Lombardia) central registry of whole population. The analysis included all 15 healthcare districts where health promotion, disease prevention, public health and socialhealthcare activities are provided and coordinated at the primary care and specialist level. Cases were assigned to each geographical area according to each patient's healthcare zone of residence at the time of the billing data in 2012. Use of the Lombardy register of prescriptions data for this study was approved by the Lombardy Data Inspection Board.

\section{Statistical analysis}

A descriptive analysis was conducted using the Point Prevalence (PP) rate (per 100,000 inhabitants) of HMV prescription (both noninvasive and tracheostomies), CPAP prescription (auto-CPAP\%, CPAP/others sleep machines \%) and LTOT prescription (liquid $\mathrm{O}_{2} \%$, oxygen gas\% and concentrators\%) in the 15 Local Health Authorities districts of Lombardy defined on the basis of billing data in 2012. For each kind of prescription (HMV, CPAP, LTOT) we defined the coefficient of variation (CV), i.e. a standardized measure of dispersion of a probability distribution expressed as a percentage, and we showed the districts of residence that were below the $5^{\text {th }}$ (P5) and above the $95^{\text {th }}$ percentile (P95) of each distribution. Data analysis and processing were performed using Prism 4, Graph Pad Software. Normality of the data was checked by Shapiro-Wilk test and correlations were performed using Pearson's correlation analysis. A p value $<0.05$ was considered statistically significant.

\section{Results}

Lombardy is an Italian Region with a varied geographical terrain comprising mountains (40.4\%), hills (12.4\%) and lowlands (47.1\%). It has a population of 10 million, ranging from $1,600,000$ in the Local Health Authority of Milan to 183,000 in the Sondrio health district. Ten percent of the population is aged 0 to 14 years. Table 1 shows Lombardy's characteristics in terms of number of residents and number of specialized respiratory units and sleep centers (Prescrition centers were for the $21.8 \%$ University or Research Center Hospitals vs $78.2 \%$ non-university hospitals and $64 \%$ public hospitals vs $36 \%$ private hospitals) [10-12].

Table 1. Number of dedicated hospital units (respiratory wards and sleep centers) and inhabitants for each Local Health Authority district in the Lombardy Region.

\begin{tabular}{|c|c|c|c|c|c|}
\hline Health districts & $\begin{array}{c}\text { Respiratory wards, } \\
\text { n }\end{array}$ & $\begin{array}{l}\text { Sleep centers, } \\
\mathbf{n}\end{array}$ & $\begin{array}{c}\text { Inhabitants, } \\
\text { n }\end{array}$ & $\begin{array}{l}\text { Inhabitants/ } \\
\text { respiratory wards }\end{array}$ & $\begin{array}{l}\text { Inhabitants/ } \\
\text { slee centers }\end{array}$ \\
\hline Milano & 24 & 9 & $1,677,000$ & 69,613 & 185,637 \\
\hline Milano 1 & 5 & 3 & 955,000 & 238,782 & 318,377 \\
\hline Milano 2 & 4 & 1 & 630,000 & 126,000 & 630,000 \\
\hline Mantova & 5 & 1 & 415,000 & 85,201 & 426,005 \\
\hline Como & 5 & 3 & 595,000 & 118,860 & 198,100 \\
\hline Varese & 10 & 2 & 883,000 & 88,773 & 443,865 \\
\hline Sondrio & 2 & 1 & 183,000 & 91,584 & 183,169 \\
\hline Brescia & 8 & 4 & $1,155,000$ & 146,296 & 292,593 \\
\hline Valle Camonica & 1 & 1 & 101,600 & 107,039 & 107,039 \\
\hline Bergamo & 6 & 2 & $1,098,000$ & 183,123 & 549,370 \\
\hline Cremona & 3 & 2 & 363,000 & 121,184 & 181,777 \\
\hline Pavia & 9 & 3 & 548,000 & 59,518 & 178,555 \\
\hline Lodi & 2 & 2 & 227,000 & 120,808 & 120,808 \\
\hline Monza & 4 & 2 & 849,000 & 212,409 & 424,818 \\
\hline Lecco & 4 & 2 & 340,000 & 85,485 & 170,971 \\
\hline
\end{tabular}


In all Local Health Authorities, there is at least 1 respiratory ward (RW) and 1. sleep center (SC) available (range 1-24 for RW and range 1-9 for SC) [10-12]. But, as can be seen from table 1, the number of specialist units available varies greatly across the Lombardy territory and is not matched to the needs of the population. The ratio of differences between districts are 1:4 for RW and 1:6 SC.

\section{HMV prescription}

In 2012, 6325 patients were on HMV. The crude rate of HMV prescriptions was $63 / 100,000$, with a high variation across districts (from $8 / 100,000$ in Milano 1 to $150 / 100,000$ in Pavia) (Figure 1A). The systematic coefficient of variation (CV) methods, which measure varia-
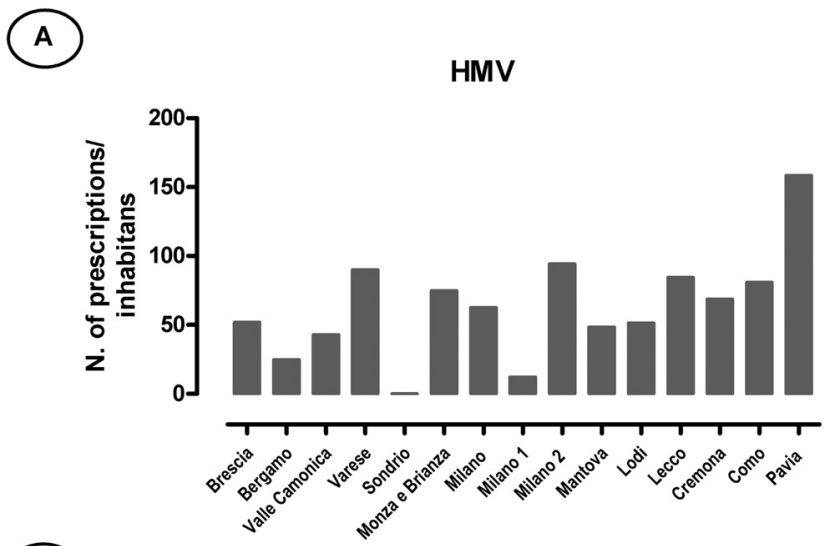

(B)

CPAP

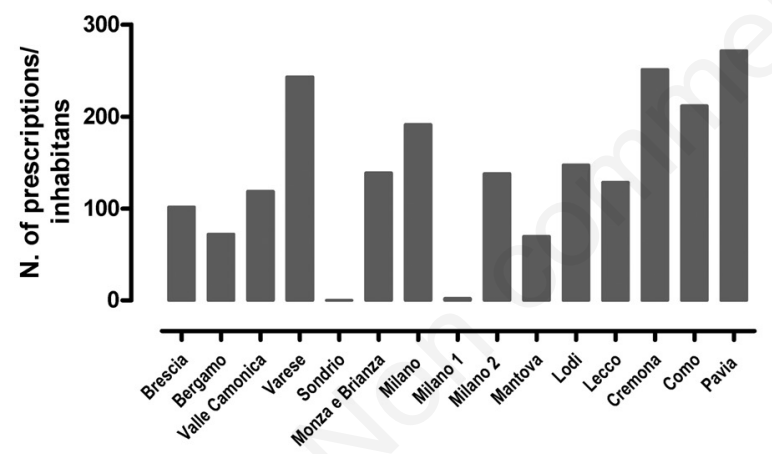

(C)

LTOT

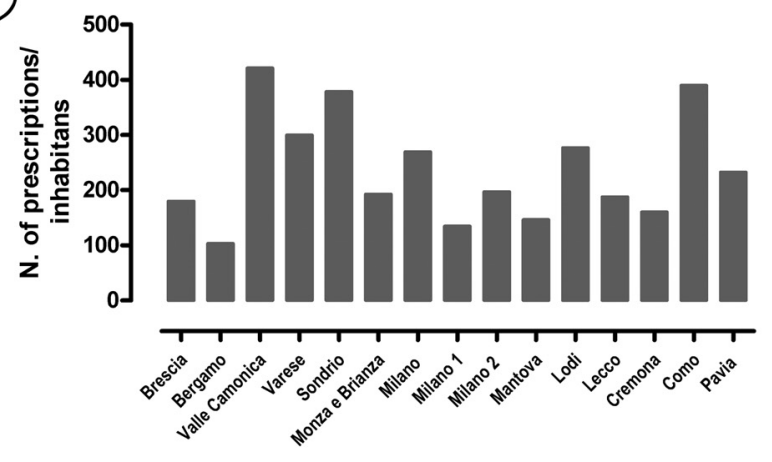

Figure 1. Prescription rate (per 100,000 inhabitants) for HMV (A), CPAP (B) and $\mathrm{O}_{2}$ (C) in the Lombardy Local Health Authority districts. HMV, home mechanical ventilation; CPAP, continuous positive airway pressure; LTOT, long-term oxygen therapy. tion not due to chance, showed a high variation in HMV [CV = 0.5187;

5-95\% confidence interval $(\mathrm{CI})=47.12-88.16$; mean 148.91; standard deviation (SD) = 77.24]. Some districts were above (Varese, Milano 2, Pavia) and some below (Milano 1, Bergamo, Valle Camonica) the area defined by the CI, corresponding to overuse or underuse of HMV with respect to the average of all the districts, respectively.

\section{CPAP prescription}

In 2012, 14,237 patients were on CPAP with a crude prescription rate of $142 / 100,000$. A high variation in Local Health Authorities districts (from 56/100,000 in Mantova to 260/100,000 in Pavia) was observed (Figure 1B). High variation in CPAP (CV = 0.60; 5-95\% CI 2.09-251.13; Mean 139; SD 83.77). Some districts were above (Cremona and Pavia) and others below (Milano 1 and Sondrio) the area defined by the confidence interval. CPAP devices were prescribed in $95.23 \%$ of cases (range 90-99.1) while auto-CPAP in $4.77 \%$ of cases (range $0.18-10$ ).

\section{LTOT prescription}

In 2012, 21,826 patients were on LTOT with a crude prescription rate of 217/100,000. A high variation in Local Health Authorities districts (from 100/100,000 in Bergamo to 410/100,000 in Valle Camonica) was observed (Figure 1C). Also for LTOT, a high intra-regional variation was observed (CV $=0.41 ; 5-95 \%$ CI 183.85-292.84; mean 238; SD 98). Some districts were above (Valle Camonica, Como, Sondrio) and others below (Milano 1, Bergamo, Brescia and Varese) the area defined by the confidence interval. Liquid oxygen was prescribed in $94 \%$ of cases (range $52-99.2$ ), oxygen concentrators only in $3.8 \%$ (range $0-17.85$ ), while in the other cases gaseous oxygen was prescribed $(2.08 \%$ as mean with a range 0-47.7).

Figure 2 shows the negative correlation between HMV (Panel A), CPAP (Panel B) and LTOT (Panel C) prescriptions and the inhabitants/respiratory wards ratio. Table 2 shows a highly significant correlation between HMV prescription and Respiratory wards, CPAP prescriptions and LTOT prescriptions showing a strong relation between supply and patients' demand and among all respiratory prescription opportunities.

\section{Discussion}

Few reports have described the prevalence and variability of HMV, CPAP and LTOT prescription. To the best of our knowledge, this is the first survey evaluating these issues in a major Region of Italy. The aim of this study was to analyze the variability in the prescription of different ventilators, CPAP and oxygen devices based on administrative data available from the public health service financial records. Variability in the clinical practice regarding HMV, CPAP and oxygen devices prescription ratio occurred across all Local Health Authorities districts. The high variation identified between districts could be explained by different factors related to supply and demand for the service.

Studies (Table 3) showing variability in the prescription of HMV in Europe have been published for over 20 years [13]. The Eurovent Survey found that HMV variability concerned both the number of prescriptions and the profile of patients for whom this treatment was prescribed, neither of which was related to the characteristics of the healthcare system or the economic situation in the country involved [1]. The national Swedish HMV register also highlighted variability in prescription within the country itself, despite a similar level of healthcare coverage $[14,15]$. In Denmark, there are only 2 centers that pre- 
scribe HMV; in this country, the prevalence of HMV is low, and the therapy is usually indicated in younger patients with neuromuscular diseases [14]. A recent study on the variability of HMV in Australia and New Zealand suggested that, in addition to variability in prevalence, HMV was more likely to be started in obese patients in New Zealand than in Australia [16].

In England [17] evidence of variability and difficulty of access to treatment led to calls for the creation of a register, and for the indications to be standardized by means of clinical practice guidelines. A crude rate of HMV prescriptions of $23 / 100,000$ was observed in
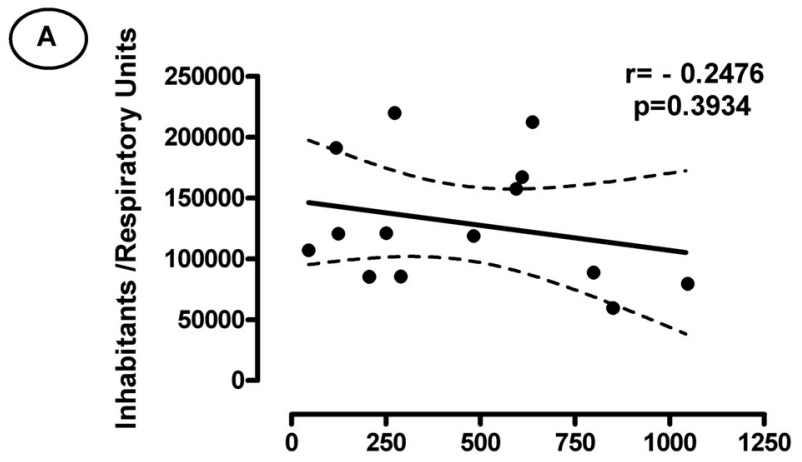

HMV prescriptions
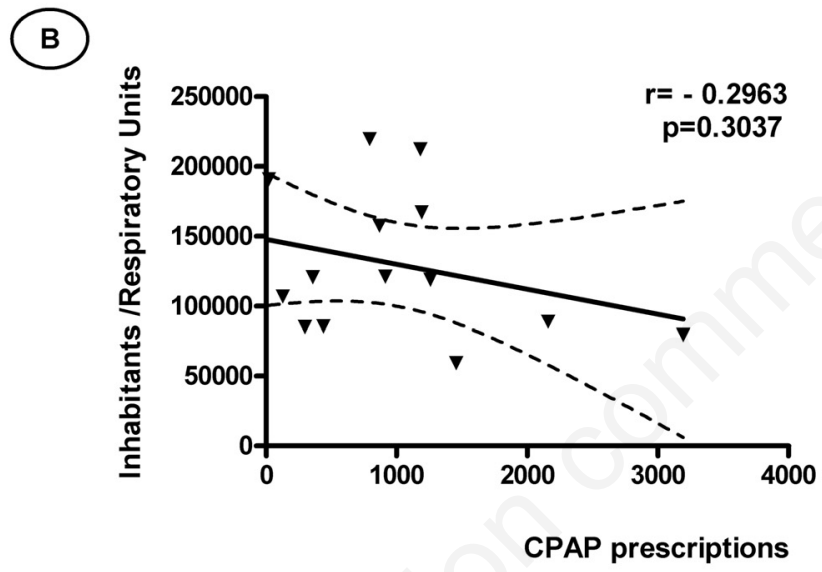

C

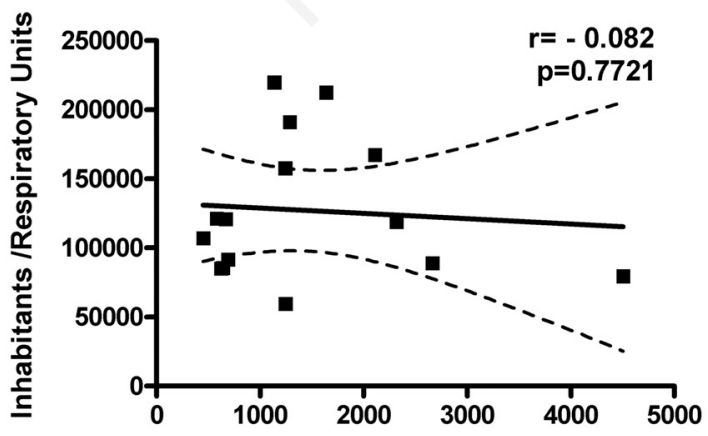

LTOT prescriptions

Figure 2. Correlations between number of inhabitants per Respiratory Unit and prescriptions for $\mathrm{HMV}(\mathrm{A}), \mathrm{CPAP}(\mathrm{B})$ and $\mathrm{O}_{2}$ (C). HMV, home mechanical ventilation; CPAP, continuous positive airway pressure; LTOT, long-term oxygen therapy.
Catalonia (Spain) [18]. Compared to other countries and to Italy as a whole [19], HMV prescription in Lombardy $(63 / 100,000)$ seems to be the highest ratio published so far in the literature (Table 3 ). The first reason for this high ratio is that the present data were obtained from crude and sure administrative data not coming from doctors' or hospitals' surveys. Although HMV is not technically complex, decision-making and resolving complications require high skills that justify concentrating the prescription in respiratory centers $[18,21]$ widely distributed among all health Lombardy districts. Another factor that may explain a high variability is the absence of objective evidence-based criteria for starting HMV, especially in obese or COPD patients [18]. On the other hand, the wide variety of diagnoses or patients potentially in need of HMV could also explain the variations observed. The attitude of physicians to ventilation seems in fact to be one of the most important factors behind the variability $[18,22]$. The high number of prescriptions in Lombardy could be related to the number of prescribing centers and their characteristics (presence of a respiratory specialist) (Table 2). We have confirmed previous data in Catalonia [18] that districts with a high number of Hospitals with a respiratory medicine unit were associated with a greater number of cases (Table 2) while the high variability in prescribing HMV in Lombardy has demonstrated inhomogeneity in the HMV care program.

Data on the prevalence of CPAP treatment for sleep apnea in different countries around the world are not clear, probably due to the mixed supply from both private market (not clearly measurable) and the public health organizational system. Only 17 individuals out of 3300 respondents (weighted prevalence $0.73 \%$ ) reported being prescribed CPAP in an Australian survey for people with OSAS [23]. In Ontario, Canada, the rate of new CPAP approvals ranged from $1.86 / 1000$ residents in 2006 to $2.93 / 1000$ in 2011 , while the annual number of diagnostic polysomnograms continued to rise from $16.1 / 1000$ in 2006 to $18.5 / 1000$ in 2013 [24]. In Catalonia [25] the reported ratio for CPAP prescription is 54:100,000 inhabitants while the estimated number of CPAP prescriptions in Italy ranged from to 44:100,000 [19] to 33:100,000 inhabitants, 200,000 [26] being the estimated number of CPAP devices used. Variability in clinical practice was found in all Lombardy Local Health Authority districts also in terms of CPAP devices prescription ratio. The high percentage of CPAP prescriptions was linked to a high sensitivity to sleep diseases throughout the entire Lombardy Health System (GPs, specialists, health payer) and to the availability of respiratory units as well as to a high number of sleep centers (Tables 1 and 2). We are confident of the crude rate of CPAP devices in Lombardy because at the time of data collection no private market for CPAP was available and all the CPAP devices were censored.

The crude rate of LTOT prescription ranges from 3.28/100,000 in USA [27] to $36 / 100,000$ in Italy [19], 48.1/100,000 in Denmark [28] and 90/100,000 in France [29] The number of respiratory units and specialists available in a particular area could cause variability in prescription, as has been observed with other respiratory therapies such as home oxygen therapy [6]. However, this phenomenon does not explain the variations within the 15 Lombardy areas, where access is easy and a large number of specialists are available. The high rate of LTOT prescription (217/100,000 inhabitants in Lombardy) is also tied to the regional funding approach which favors a prescriptive option for border-line chronic respiratory failure (night-time and during exercise). In some areas of Lombardy, the prescriptive oxygen option may have replaced that for CPAP or HMV due to the lesser number of specialized units available.

A strength of this study is the type of analysis performed, based on available administrative data, which provides a good complement to 
clinical experience and cross-sectional studies, giving a comprehensive view of the devices' prescription problem.

The greatest limitation of this study is the lack of access to diagnoses and clinical data with the difficulty to explain the dramatic differences in prevalence of HMV between different districts and the possibility to compare present data with other reports of literature in which diagnosis was present. Nevertheless, we have previously discussed that the use of administrative data (the case of this paper) only allowed access, for reasons of data confidentiality, to the type of treatment subsidized by the public health system and zone of residence. Moreover, obtaining information from the prescribing center with a survey (which would give more detailed information on diagnosis and the technical characteristics of the treatment) might not necessarily give an idea of the population impact (a center may prescribe ventilation to patients from various geographical districts).

Unfortunately our data coming from "crude administrative data" are aggregate data according to single Health District Zone: for this reason we have not access to single center data allowing specific and details correlation between center and level of HMV prescription.

As further limitation of the study, it is important to remember that Hospitals did not begin to prescribe HMV and CPAP simultaneously. Supply companies can influence the variability of respiratory devices prescription. However, given the characteristics of the funding system, with very stringent limits on increases in prescriptions, it is unlikely that these companies have a direct effect on such prescriptions [18]. Another possible confounding factor is that some people may migrate from their district to another one to receive support and prescription.

\section{Conclusions}

The crude rate of HMV prescriptions in Lombardy is very high. The high variability found among the Lombardy Local Health Authority districts in prescribing HMV, CPAP and LTOT can be explained, in part, by the attitude of professionals toward treatment and the high availability of specialist centers with a respiratory unit dedicated to HMV and sleep study. The wide differences observed can help to identify areas characterized from a reduced availability of instruments and structure for diagnosis and therapy of respiratory diseases requiring home mechanical ventilation. The rate of HMV prescription described in the literature is probably underestimated. Analysis of administrative data and variability mapping can help identify unexplained variations, i.e. areas of reduced access, so leading to an improved standardization of healthcare services. The expected action coming from the Health Payer is to propose an Audit among prescribers to interpret the described huge variability.

Table 2. Correlations between respiratory units and HMV, CPAP and LTOT prescriptions.

\begin{tabular}{|c|c|c|c|}
\hline & HMV prescription, n & CPAP prescription, $\mathrm{n}$ & LTOT prescription, n \\
\hline \multicolumn{4}{|l|}{ Respiratory units, n } \\
\hline Pearson $r$ & 0.8023 & 0.8916 & 0.8987 \\
\hline $95 \%$ confidence interval & 0.4731 to 0.9349 & 0.6850 to 0.9655 & 0.7162 to 0.9662 \\
\hline P value (two-tailed) & 0.0006 & $P<0.0001$ & $P<0.0001$ \\
\hline \multicolumn{4}{|l|}{ HMV prescription, $n$} \\
\hline Pearson $\mathrm{r}$ & 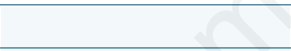 & 0.9120 & 0.8002 \\
\hline $95 \%$ confidence interval & 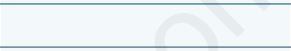 & 0.7390 to 0.9722 & 0.4684 to 0.9342 \\
\hline P value (two-tailed) & $a^{2}+x^{2}$ & $P<0.0001$ & 0.0006 \\
\hline CPAP prescription, $n$ & 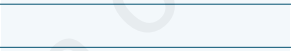 & & \\
\hline Pearson $\mathrm{r}$ & 8 & & 0.9048 \\
\hline 95\% confidence interval & +8 & & 0.7197 to 0.9698 \\
\hline $\mathrm{p}$ value (two-tailed) & & & $P<0.0001$ \\
\hline
\end{tabular}

HMV, home mechanical ventilation; CPAP, continuous positive airway pressure; LTOT, long-term oxygen therapy.

Table 3. Comparison of the crude rate of HMV prescription (per 100,000 inhabitants) in Lombardy Region, Italy. Comparison with available international data.

\begin{tabular}{lcc} 
& Year of survey & HMV prescriptions $\mathbf{1 0 0 , 0 0 0}$ \\
Lombardy (this study) & 2012 & 63 \\
\hline Italy [19] & 2010 & 20 \\
\hline EU [1] & 2004 & 6.6 \\
\hline Catalonia (Spain) [18] & 2011 & 23 \\
\hline France [20] & 2009 & 32 \\
\hline Canada [21] & 2014 & 12.9 \\
\hline New Zealand [16] & 2012 & 12 \\
\hline Australia [16] & 2012 & 9.9 \\
\hline Denmark [14] & 1996 & 5.5 \\
\hline Sweden [14,15] & 1996 & 6.1 \\
\hline
\end{tabular}

HMV, home mechanical ventilation. 


\section{References}

1. Lloyd-Owen SJ, Donaldson GC, Ambrosino N, et al. Patterns of home mechanical ventilation use in Europe: results from the Eurovent survey. Eur Respir J 2005;25:1025-31.

2. Kohnlein T, Windisch W, Kohler D, et al. Non-invasive positive pressure ventilation for the treatment of severe stable chronic obstructive pulmonary disease: a prospective, multicentre, randomised, controlled clinical trial. Lancet Respir Med 2014;2:698-705.

3. MacIntyre EJ, Asadi L, Mckim DA, Bagshaw SM. Clinical Outcomes Associated with Home Mechanical Ventilation: A Systematic Review. Can Respir J 2016;2016:6547180.

4. King AC. Long-term home mechanical ventilation in the United States. Respir Care 2012;57:921,30; discussion 930-2.

5. Farre R, Lloyd-Owen SJ, Ambrosino N, et al. Quality control of equipment in home mechanical ventilation: a European survey. Eur Respir J 2005;26:86-94.

6. Williams BT. Geographical variations in the supply of domiciliary oxygen. Br Med J (Clin Res Ed) 1981;282:1941-3.

7. Wennberg J, Gittelsohn. Small area variations in health care delivery. Science 1973;182:1102-8.

8. Haynes RB, Devereaux PJ, Guyatt GH. Physicians' and patients' choices in evidence based practice. BMJ 2002;324:1350.

9. Amaducci S, Clini E, Sturani C, Ambrosino N, Galavotti V, Vitacca M. Home- care (HC) management in chronic hypercapnic respiratory failure (CHRF): preliminary results from an experience in Lombardy. Eur Respir J 1996;9(Suppl 23); 422S.

10. [Internet]. [2017]. Available from: http://www.asr-lombardia.it/ASR/. Accessed June 14, 2017.

11. [Internet]. [2016]. Available from: http://www.aiponet.it/. Accessed June 14, 2017.

12. [Internet]. [2016]. Available from: http://www.ats-milano.it/ITA /Homepage.aspx. Accessed June 14, 2017.

13. Fauroux B, Howard P, Muir JF. Home treatment for chronic respiratory insufficiency: the situation in Europe in 1992. The European Working Group on Home Treatment for Chronic Respiratory Insufficiency. Eur Respir J 1994;7:1721-6.

14. Midgren B, Olofson J, Harlid R, et al. Home mechanical ventilation in Sweden, with reference to Danish experiences. Swedish Society of Chest Medicine. Respir Med 2000;94:135-8.
15. Laub M, Berg S, Midgren B, Swedish Society of Chest Medicine. Home mechanical ventilation in Sweden-inequalities within a homogenous health care system. Respir Med 2004;98:38-42.

16. Garner DJ, Berlowitz DJ, Douglas J, Harkness N, Howard M, McArdle N, Naughton MT, Neill A, Piper A, Yeo A, Young A. Home mechanical ventilation in Australia and New Zealand. Eur Respir J 2013;41:39-45.

17. Garner DJ, Berlowitz DJ, Douglas J, et al. Provision of home mechanical ventilation and sleep services for England survey. Thorax 2013;68:880-1.

18. Escarrabill J, Tebe C, Espallargues M, et al. Variability in home mechanical ventilation prescription. Arch Bronconeumol 2015;51:490-5.

19. Garuti G, Galavotti V, Vitacca M. Tipologia e Prevalenza dei Servizi di Assistenza Domiciliare in pazienti affetti da Insufficienza Respiratoria Cronica: Survey nazionale AIPO. Rassegna di Patologia dell'Apparato Respiratorio 2017; 2: in press.

20. Escarrabill J. Organisation and delivery of home mechanical ventilation. Breathe 2009;6:36-42.

21. Rose L, McKim DA, Katz SL, et al. Home mechanical ventilation in Canada: a national survey. Respir Care 2015;60:695-704.

22. Dybwik K, Tollali T, Nielsen EW, Brinchmann BS. Why does the provision of home mechanical ventilation vary so widely? Chron Respir Dis 2010;7:67-73.

23. Marshall NS, Bartlett DJ, Matharu KS, et al. Prevalence of treatment choices for snoring and sleep apnea in an Australian population. J Clin Sleep Med 2007;3:695-9.

24. Povitz M, Kendzerska T, Hanly PJ, et al. Profile of continuous positive airway pressure treated patients in Ontario, Canada, 20062013: A population-based cohort study. In: C80-D. Sleep and health policy. Am Thoracic Soc 2017. p. A6527.

25. [Internet]. [2016]. Available from: http:/observatorisalut.gencat.cat/ca. Accessed June 14, 2017.

26. [Internet]. [2016]. Available from: http://www.apneedelsonno.it/ Accessed June 14, 2017.

27. Kim V, Benditt J0, Wise RA, Sharafkhaneh A. Oxygen therapy in chronic obstructive pulmonary disease. Proceedings of the American Thoracic Society 2008;5:513-8.

28. Ringbaek TJ, Lange P. Trends in long-term oxygen therapy for COPD in Denmark from 2001 to 2010. Respir Med 2014;108:511-6.

29. Fuhrman C, Boussac-Zarebska M, Roche N, Delmas MC. Long-term oxygen therapy in France, 2006-2011. Rev Mal Respir 2014:31:421-9. 